\title{
Etat de la sécurité laser en France
}

\author{
F. SANCHEZ*
}

\section{RÉSUMÉ}

\begin{abstract}
A travers une expérience acquise lors de l'animation de stages de sécurité "laser" depuis plusieurs années, nous dressons le bilan des problèmes non ou mal résolus qui se posent aux utilisateurs de lasers en France. Sont abordés, en particulier, les problèmes de langage, de matériel, de service après-vente, de surveillance médicale, de formation initiale.
\end{abstract}

\section{ABSTRACT}

Through the experience gained after several years of laser safety courses we give a list of unsolved problems that are set to French laser operators, viz language, material, after-sales service, medical surveillance, initial training.

\section{INTRODUCTION}

Le présent rapport a pour objet d'une part, de préciser l'état actuel de la sécurité laser en France, et d'autre part, de souligner les problèmes non ou mal résolus.

II se fonde sur une expérience acquise depuis quatre années en "Formation permanente" (stages de sécurité "Laser" organisés par le CEN de Saclay), où un grand nombre de témoignages et d'études de cas "sur le terrain" (en particulier plusieurs centres du CEA) ont été recueillis. La genèse, le contenu et les premiers développements de ces stages ont été décrits par ailleurs. Cette expérience est complétée par le travail sur laser avec les élèves-ingénieurs de P'Ecole supérieure d'optique depuis 1969, ainsi que les contacts pris avec mes collègues de l'Université de Paris XI (Centre d'Orsay). En particulier, doit être soulignée l'aide apportée par les membres (de diverses provenances) de la récente commission "Laser" de Saclay.

Les positions prises dans le présent article n'engagent que leur auteur : elles sont faites indépendamment de toute pression extérieure, et sans considération quelconque d'intérêt personnel.

L'établissement de ce rapport répond à un devoir déontologique visà-vis du Commissarait à l'énergie atomique (CEA) qui m'accorde sa confiance. II est d'autant plus nécessaire qu'on assiste à une accélération du nombre

\footnotetext{
* Université de Paris Sud et Ecole supérieure d’optique, BP 43, 91406 Orsay Cedex.
} 
d'accidents rétiniens. II s'agit d'une situation typique de crise où il y a un manque d'adéquation technique - utilisation, exacerbée par l'absence de réglementation en France, alors qu'elle existe depuis huit ans aux USA (annexe I).

\section{PROBLĖMES LIÉS AU LANGAGE}

Les problèmes de langage sont énormes et parfois insoupçonnés. Cela est particulièrement vrai en sécurité "Laser" où nombre de documents sont traduits de l'anglais (annexe IV).

Un principe de base qui se dégage de l'expérience acquise lors des stages est qu'il faut refuser l'inféodalisation à la fois aux terminologies et aux mentalités étrangères :

- Un exemple sur la terminologie : le mot " avoid" ne doit pas être traduit par "éviter", mot beaucoup trop faible.

- Un exemple de tournure d'esprit américaine : les tableaux des EMA (expositions maximales admissibles) comportent des erreurs pédagogiques graves, lesquelles sont reproduites telles quelles dans la traduction de l'INRS de 1982 (annexe I). Ces erreurs rendent très difficile l'emploi correct de ce document. Ainsi, 9 personnes sur 10 se trompent de tableau pour le calcul de l'EMA en lumière diffuse ponctuelle. D'autre part, on note l'absence d'une formule concentrée et non-ambiguë pour le calcul, si important, de l'EMA pour un laser pulsé. Par conséquent, il est urgent de reformuler ce document de manière logique et pratique.

Dans le même ordre d'idée, il faut exiger des importateurs et des constructeurs français (!) des notices en bon français. Ces notices devraient comporter des exemples de calcul d'EMA pour des situations simples, ainsi que des prescriptions élémentaires de sécurité. Nous considérons qu'il s'agit là d'un élément-clé de la sécurité "Laser". Exemple significatif : des lasers suisses (marque Lasag) de type YAG (petit usinage), à haut risque rétinien, sont fournis avec des notices en allemand!

\section{PROBLĖMES LIÉS AU MATÉRIEL}

Pour les constructeurs, de manière générale, la sécurité ne se vend pas. C'est donc aux acheteurs de matériel laser qu'il revient de faire jouer la concurrence entre les constructeurs dans la direction d'un alignement soit sur les normes existant aux USA (BHR 1976), soit sur les prescriptions de fabrication en cours de publication par la CEI (texte français) (cf. annexe 1). Ceci ne s'applique pas aux lasers médicaux, pour lesquels le ministère de la Santé a fait établir un protocole d'essais d'homologation, s'appuyant sur la norme NF 74-300 "Appareils d'électricité médicale" édité par l'UTE (30 juin 1963).

II semble qu'un problème important n'ait pas été envisagé : un laser peut émettre des longueurs d'onde parasites beaucoup plus dangereuses 
que le rayonnement nominal. C'est, en particulier, le cas des lasers UV pulsés qui peuvent émettre dans l'infrarouge proche, d'où un important risque rétinien. Les prescriptions à venir devraient obliger les constructeurs à considérer ce point si méconnu. La classification BRH des rayonnements des appareils à laser (annexe II), qui est reconduite par la CEI, est nettement insuffisante pour les lasers pulsés à risque rétinien. En effet, avec un laser continu d'1 W, on se trouve 1000 fois au-dessus de I'EMA en exposition brève $(0,25 \mathrm{~s})$, tandis que pour un laser pulsé de puissance moyenne d' $1 \mathrm{~W}$, on peut se trouver $10^{6}$ fois au-dessus de l'EMA. Et pourtant, ces lasers sont situés dans la même classe (classe 4). Le caractère extrêmement dangereux de ces lasers pulsés à risque rétinien explique l'accélération du nombre d'accidents, en particulier dans le domaine visible, où il n'existe généralement pas de protecteurs oculaires à haut rendement lumineux.

Des signaleurs "Danger Laser" sont commercialisés, mais ne peuvent prétendre à une totale efficacité en l'absence d'homologation.

\section{SERVICE APRĖS-VENTE. L'INTERVENTION}

De nombreux participants aux stages ont remarqué l'extrême désinvolture de certains réparateurs de matériel laser. Rappelons qu'il y a eu un accident mortel en France sur l'alimentation d'un laser $\mathrm{CO}_{2}$. Ce comportement est extrêmement préjudiciable à la sécurité des personnels, soit directement, soit surtout indirectement, car on peut avoir tendance à copier les gestes du soi-disant "spécialiste", ce qui peut contribuer à une anti-formation importante.

L'organigramme de l'annexe III rappelle l'idéal vers lequel devrait tendre toute installation à laser : le régime de routine, c'est-à-dire l'élimination totale du risque dans le cadre d'une procédure d'utilisation définie. Lorsqu'on sort de ce cadre (réparation, modification), on passe en régime d'intervention, terme emprunté à la sécurité électrique, qui implique une étude de minimalisation du risque dont ne saurait se dispenser même les utilisateurs d'un appareil à laser de classe 1 (souvent des systèmes industriels). II est donc illusoire de tenter une approche différente de la sécurité laser selon que le travail est du type reproductif (industriel) ou évolutif (recherche).

Ainsi, les chercheurs, tout comme les prestateurs de service aprèsvente, operent en intervention permanente, ce qui implique une formation poussée à la sécurité laser. Concernant le service après-vente, un moyen simple de faire se modifier de façon positive la situation serait d'assujettir l'achat d'un laser de la classe de rayonnement 4 à la production par le constructeur d'une attestation de "suivi de stage sécurité laser" pour les prestateurs de service après-vente qu'il s'engage à mettre à la disposition du client.

\section{ASPECTS MÉDICAUX}

En l'absence d'enquête épidémiologique systématique (ce n'est que récemment, sur l'initiative du CEA, que les accidents laser ont commencé 
à être répertoriés comme tels par la sécurité sociale), on ne peut qu'être très prudent sur les effets à très long terme (plusieurs années). En effet, il nous a semblé avoir remarqué sur nous-mêmes une augmentation importante de la sensibilité à l'éblouissement pour des doses de rayonnement diffusé par des taches ponctuelles (cas très fréquent) inférieures à l'EMA journalier. Le même état d'éblouissement se produit rapidement en conduite nocturne à la lumière des codes ou des phares d'autres voitures. Cette observation personnelle a été corroborée par maints témoignages. II semble que cette sensibilité à l'éblouissement varie fortement avec l'âge et les individus. En état d'éblouissement, on se trouve incapable de lire ou de regarder la télévision dans la soirée, mais le lendemain il n'y a aucune séquelle apparente. En outre, nous avons noté sur nousmêmes une gêne visuelle excessive pour les luminances intermédiaires entre la vision photopique (jour) et la vision scotopique (nuit).

II semble que les ophtalmologues n'aient pas connaissance de ce type de pathologie. Des recherches dans ce sens devraient être effectuées. Dans l'immédiat, on ne peut que recommander aux utilisateurs de laser d'exercer une auto-surveillance de leur vision.

Si le problème de la surveillance médicale est loin d'être résolu, il faut insister sur l'importance cruciale d'écarter des lasers les personnes n'ayant qu'un œil en bon état (un accident récent a été signalé chez une stagiaire dans une entreprise de construction de lasers, lœil atteint étant l’il valide). Une réglementation à ce sujet est urgente.

\section{CONCLUSIONS}

La grande variété des lasers et de leurs applications fait que la connaissance et l'utilisation des EMA est indispensable, si l'on ne veut pêcher ni par excès, ni par défaut dans l'évaluation des risques. Pour des expositions non chroniques, il semble qu'il faille leur accorder une certaine confiance, les valeurs actuelles ayant été peu retouchées par rapport aux valeurs données dans I'ANSI, 1976 (annexe I). Par contre, pour des expositions chroniques, le problème reste entier. II s'ensuit qu'il ne faut pas laisser vieillir des équipes d'expérimentateurs autour de matériels à laser dans les laboratoires de recherche. En dehors du fait que ce type de travail est spécialement pénible, c'est, de toute façon, un principe général vis-à-vis de la sécurité, spécialement si plusieurs types de risque sont simultanément présents.

Un problème de fond concerne la formation initiale des jeunes diplômés, pour lesquels la formation permanente paraît avoir moins d'impact que pour les personnels déjà expérimentés.

Dans le cadre du renouveau prévu de la pédagogie permettant une meilleure adéquation études-vie professionnelle, les questions de sécurité (électrique, chimique, rayonnement,...) doivent être abordées assez tôt dans le cycle d'enseignement.

Enfin, il semble qu'il ne faille pas trop compter sur une réglementation en France dans un avenir proche, principalement en raison du fait qu'une telle réglementation doit passer par le niveau européen d'abord ; c'est pourquoi il ne faut pas attendre pour utiliser les idées dégagées dans le présent article. 
ETAT DE LA SECURITE LASER EN FRANCE

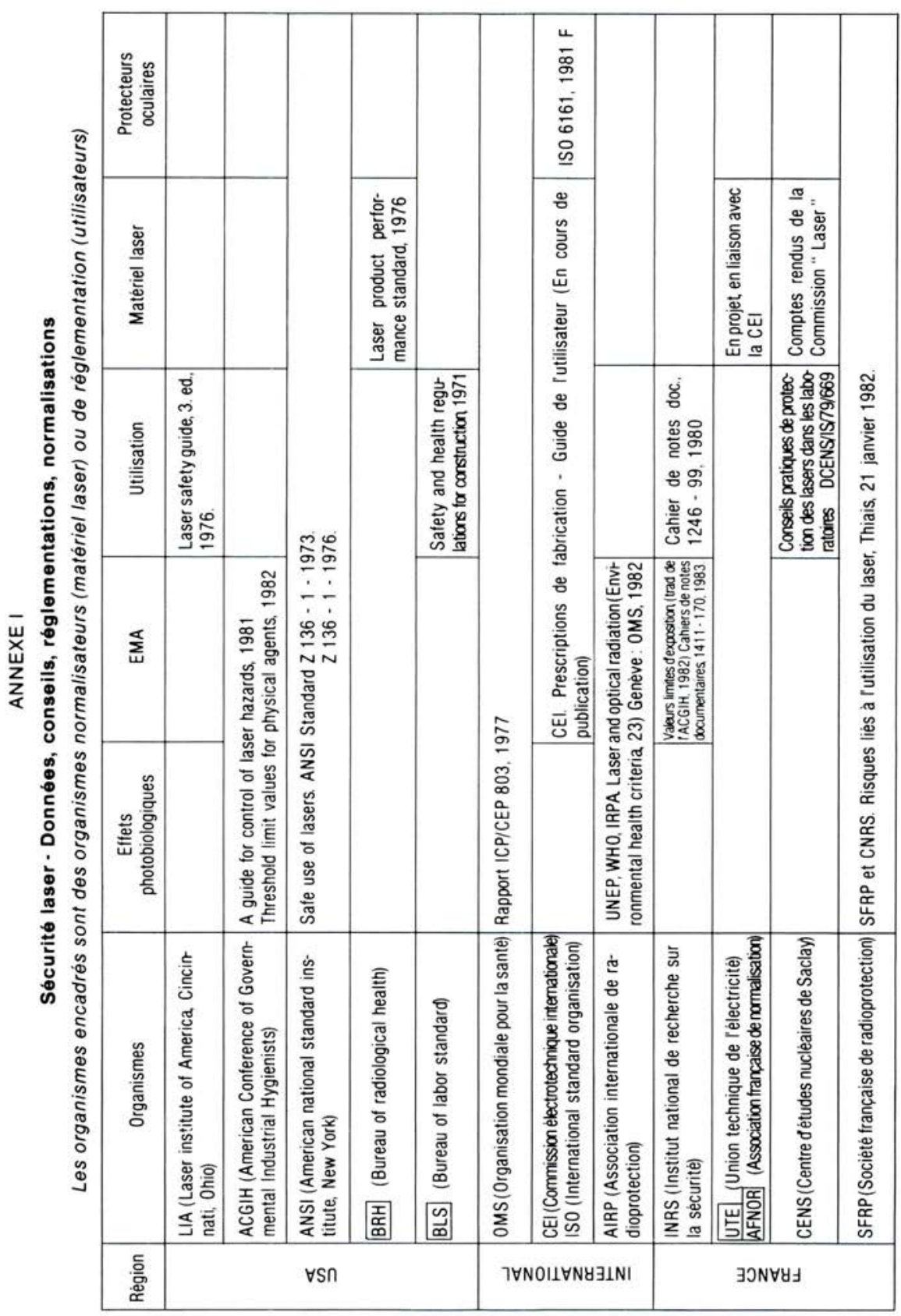




\section{Classes de rayonnement des lasers et appareils à laser}

\section{A. JACQUEMMOZ, F. SANCHEZ}

Conformément à la norme américaine BHR (août 1976), les fabricants de lasers ou d'appareils à laser sont tenus aux USA de classer leurs appareils destinés à la vente aux utilisateurs directs, en fonction du risque faisceau que peuvent présenter les appareils lors d'une utilisation normale, en particulier sans bter de capot de protection. Le classement s'opère en fonction de l'émission accessible, c'est-à-dire le rayonnement sortant effectivement de Pappareil à laser ; cette émission accessible étant comparée à des limites d'émission accessible (LEA) qui délimitent les classes.

Une norme équivalente est envisagée en France, avec des valeurs de LEA révisées par la Commission électrotechnique internationale (CEI) en juillet 1982.

L'intérêt de ces classes est pour l'utilisateur. En effet, il peut définir immédiatement, au seul vu de la classe (sauf pour la classe 4) des comportements sans risque (dans l'état actuel des connaissances médicales), et ceci sans avoir à recourir à des mesures radiométriques et des comparaisons avec les tableaux d'EMA (exposition maximale admissible).

Définitions : $t$ : temps d'exposition

d: distance observateur-écran.

\begin{tabular}{|c|c|c|c|}
\hline Classe $\begin{array}{r}\text { Type } \\
\text { dexpo- } \\
\text { sition }\end{array}$ & $\begin{array}{c}\text { Exposition accidentelle } \\
\text { directe (ou réflexion } \\
\text { spéculaire) }\end{array}$ & $\begin{array}{l}\text { Exposition à une } \\
\text { réflexion diffuse sur } \\
\text { un écran }\end{array}$ & $\begin{array}{l}\text { Objet inflammable } \\
\text { (incendie) }\end{array}$ \\
\hline 1 & \multicolumn{3}{|c|}{ SANS RISQUE } \\
\hline $\begin{array}{l}2 \\
(400-700 \mathrm{~nm})\end{array}$ & $\begin{array}{l}\text { Sans risque si : } \\
\mathrm{t}<0,25 \mathrm{~s} \\
\text { (ne pas lutter contre } \\
\text { le réflexe palpébral) }\end{array}$ & \multicolumn{2}{|c|}{ SANS RISQUE } \\
\hline $3 A$ & $\begin{array}{l}\text { Sans risque si : } \\
\mathrm{t}<0,25 \mathrm{~s} \text { et } \text { non } \\
\text { utilisation d'instru- } \\
\text { ment d'optique. }\end{array}$ & \multicolumn{2}{|c|}{ SANS RISQUE } \\
\hline 3в & DANGER & $\begin{array}{l}\text { Sans risque si : } \\
t<10 \mathrm{~s} \\
\text { et } \\
d>13 \mathrm{~cm}\end{array}$ & SANS RISQUE \\
\hline 4 & \multicolumn{3}{|c|}{ DANGER } \\
\hline $\begin{array}{l}\text { N.B. : Sa } \\
\text { des rayon }\end{array}$ & $\begin{array}{l}\text { pour la classe } 2 \text {, les } \\
\text { ements laser : } 200 \text { à } 1\end{array}$ & $\begin{array}{l}\text { asses sont définies } \\
\mathrm{nm} \text {. }\end{array}$ & s tous les \\
\hline
\end{tabular}


ANNEXE III

Sécurité laser - Organigramme de prévention

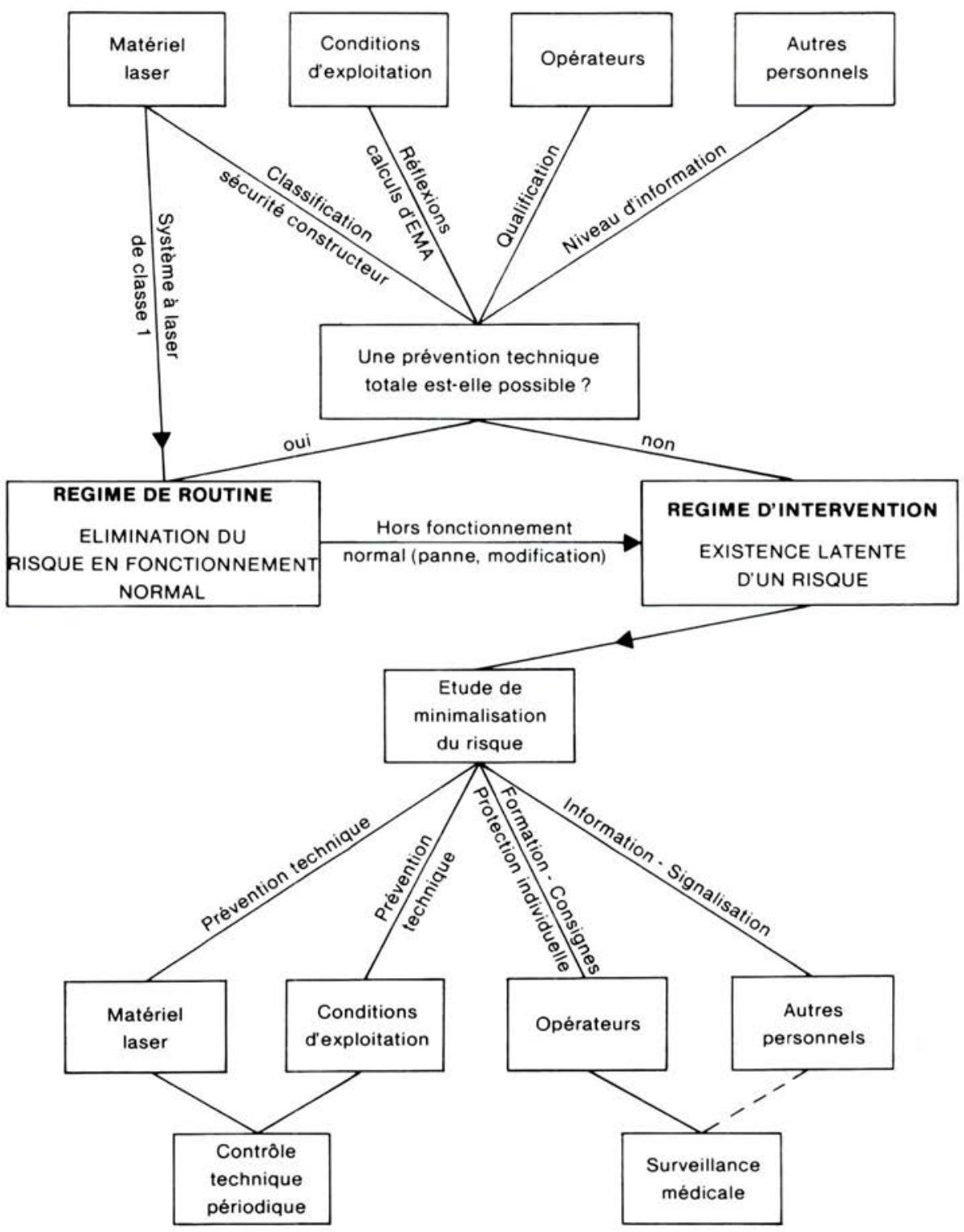




\section{ANNEXE IV}

\section{Note sur les unités photométriques}

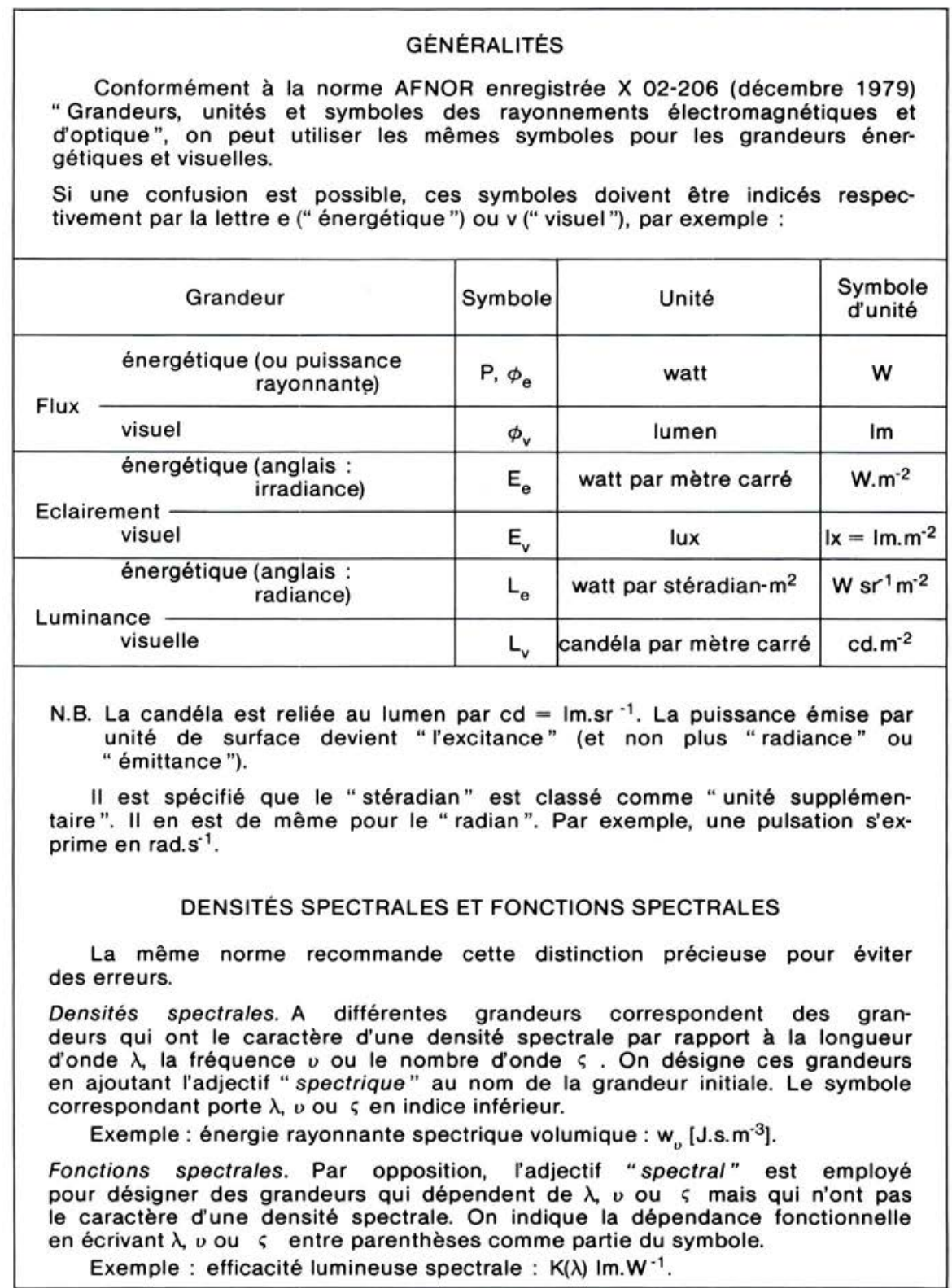

